\title{
Does Anastomosis Configuration Influence Long-term Outcomes in Patients With Crohn Disease?
}

\author{
Parajuli Anuj, Yong Sik Yoon ${ }^{1}$, Chang Sik Yu ${ }^{1}$, Jong Lyul Lee ${ }^{1}$, Chan Wook Kim ${ }^{1}$, In Ja Park ${ }^{1}$, \\ Seok-Byung Lim ${ }^{1}$, Jin Cheon Kim ${ }^{1}$ \\ Department of Surgery, Kathmandu Medical College and Teaching Hospital, Kathmandu, Nepal; \\ ${ }^{1}$ Department of Surgery, University of Ulsan College of Medicine, Asan Medical Center, Seoul, Korea
}

Purpose: In this study, we evaluated the role of various anastomoses in surgical recurrence for patients with Crohn disease (CD).

Methods: We analyzed data retrospectively from consecutive laparotomy cases involving complicated CD between 1991 and 2008. Clinical data were compared in terms of reoperation-free survival (RFS) according to the types of anastomoses, the materials used for the anastomoses, and the operating surgeon.

Results: Of 233 patients with entero-enteric or entero-colic anastomoses, 199 (85\%), 11 (5\%), and 23 (10\%) experienced side-to-side (SS), side-to-end (SE), and end-to-end (EE) anastomoses, respectively. The SS group had the following characteristics: more extensive bowel involvement, frequent obstruction, and greater stapler use; the SS anastomoses were also frequently made by specialized surgeons $(\mathrm{P}<0.001-0.004)$. EE anastomoses were frequently made by general surgeons using a hand-sewing technique $(\mathrm{P}<0.001)$. No differences in RFS were noted among the 3 groups according to the type of anastomosis and the operating surgeon. However, the hand-sewn group showed better RFS than the stapler group ( $\mathrm{P}=0.04)$.

Conclusion: The roles of the anastomotic configuration, the material used, and the operating surgeon were not significantly correlated with reoperations or complications in our retrospective CD cohort, irrespective of the higher risk of anastomosis site stricture for EE anastomoses.

Keywords: Crohn disease; Inflammatory bowel diseases; Surgery; Treatment outcome

\section{INTRODUCTION}

Crohn disease (CD) is a chronic inflammatory gastrointestinal disorder with a heterogeneous clinical course and a high incidence of recurrent flare-ups and related complications. The prediction of recurrence and related complications may be difficult to individualize. Although surgery in these patients is not curative, an estimated $80 \%$ of patients with CD may require surgery

Received: May 9, 2017 - Accepted: June 12, 2017

Correspondence to: Yong Sik Yoon M.D.

Department of Surgery, University of Ulsan College of Medicine, Asan

Medical Center, 88 Olympic-ro 43-gil, Songpa-gu, Seoul 05505, Korea

Tel: +82-2-3010-5318, Fax: +82-2-3010-6701

E-mail:yoonys@amc.seoul.kr

(c) 2017 The Korean Society of Coloproctology

This is an open-access article distributed under the terms of the Creative Commons Attribution NonCommercial License (http://creativecommons.org/licenses/by-nc/4.0) which permits unrestricted non-

commercial use, distribution, and reproduction in any medium, provided the original work is properly cited. during their lifetimes [1]. Symptomatic recurrence after 1 year of surgery is around $20 \%-30 \%$, and $20 \%$ of the patients with recurrence 5 years or longer after surgery will require reoperation $[2,3]$. The determinants of disease recurrence remain unclear, but various prognostic factors that may potentially influence the recurrence of $\mathrm{CD}$ have been isolated: age at onset, duration of $\mathrm{CD}$ before surgery, history of smoking, family history of $\mathrm{CD}$, anatomical site, medical treatment, indications for surgery (perforating and nonperforating), length of resection, anastomosis technique, residual disease in the margin, presence of granulomas in the specimen, perioperative blood transfusion, and postoperative complications. These all have variable impacts on disease recurrence, but none, apart from smoking, has established significance [4-6].

Recently, the effects of interests in the intraoperative strategies, such as changing the anastomotic configuration on the postoperative recurrence of $\mathrm{CD}$, have been investigated. The results seem to be controversial, with some authors suggesting that better results can be obtained with a side-to-side (SS) anastomosis, i.e., 
lower incidence of symptomatic recurrence and reoperations [79], while others claim that no differences exist $[5,10,11]$. A narrow anastomosis has been hypothesized to lead to fecal stasis and bacterial overgrowth, resulting in early disease recurrence [12]. This finding might lead surgeons to prefer a wide anastomosis to a narrow one. We investigated the influence of anastomotic configuration on the incidence of reoperation in patients undergoing intestinal resection for complications of CD.

\section{METHODS}

Data from a prospectively maintained database were retrospectively collected from January 1991 to December 2008. In all, 269 consecutive laparotomies for complicated CD were included in this study. Laparotomies performed for indications other than $\mathrm{CD}$, surgery without anastomosis (usually end stoma), and surgery with colo-colic anastomosis only were excluded $(n=36)$. In total, 233 cases were analyzed. Demographics, indications for surgery, location of resection, reoperation, reconstruction method, surgeon involved, and clinical complications were reviewed. In cases of reoperation, inflammation around the anastomosis and the anastomotic stricture was checked. Clinical follow-up was performed by the treating surgeons and gastroenterologists 2 weeks after discharge. Thereafter, the patients visited the gastroenterologists at 1- to 2-month intervals, depending on their status and the severity of the disease. Surgeries not to treat CD exacerbation were excluded from the reoperation data (e.g., adhesiolysis or stoma closure only). The study protocol was approved by the Institutional Review Board of Asan Medical Center (approval number: 2017-0088). Median follow-up was 93 months (range, 0-276 months).

Before 2002, all surgeries were performed by general surgeons (fellows) and colorectal surgeons (staffs), but later most inflammatory bowel disease (IBD) surgeries were performed by colorec- tal surgeons specializing in IBD. Thus, operating surgeons were classified as general surgeons, colorectal surgeons, or IBD surgeons. Anastomosis configurations were classified as end-to-end (EE), end-to-side (ES), and SS, involving entero-enteric or enterocolic anastomoses. Anastomotic materials were divided into stapler and hand-sewn. Bowel resection was done in the normal fashion with a resection margin of $2-5 \mathrm{~cm}$ on either side, if possible. Reconstruction of the resected segment was performed with a stapler (mostly linear staples) or in a hand-sewn fashion, based on the surgeon's preference. In cases with multiple reconstructions, the anastomosis of the main lesion was evaluated.

Continuous variables in each group were compared using the ttest or Wilcoxon rank sum test and are expressed as means \pm standard deviations; categorical variables were compared using the chi-square test or Fisher exact test and are expressed as frequencies (percentages). The cases were compared according to type of anastomosis, surgeon, and material used for reconstruction. Reoperation-free survival (RFS) was used to evaluate clinical outcomes and was calculated using the Kaplan-Meier method; comparisons of the RFS were done using log rank tests. P-values < 0.05 were considered statistically significant for all analyses, and all calculations were carried out using IBM SPSS Statistics ver. 21.0 (IBM Co., Armonk, NY, USA).

\section{RESULTS}

Table 1 lists the clinical features of the 233 cases of complicated CD. The majority of cases $(n=199,85 \%)$ were in the SS group. Compared to the other groups, the patients in the SS group had more extensive bowel involvement (ileo-colic type), were frequently operated on due to obstruction, received more staples, and were more frequently operated on by specialized surgeons $(\mathrm{P}<0.001-0.004$, Table 1). On the other hand, the patients in the EE group had more peritonitis, frequently received a hand-sewn

Table 1. Clinical characteristics of the surgically-treated Crohn disease patients in the current study series

\begin{tabular}{|c|c|c|c|c|c|}
\hline Characteristic & Total $(n=233)$ & SS $(n=199,85 \%)$ & SE $(n=11,5 \%)$ & $\mathrm{EE}(\mathrm{n}=23,10 \%)$ & P-value \\
\hline Male sex & $169(73)$ & $140(70)$ & $10(91)$ & $19(83)$ & 0.173 \\
\hline Mean age (yr) & 29.9 & 30.0 & 28.2 & 29.3 & 0.834 \\
\hline Reoperation & $50(22)$ & $44(22)$ & $2(18)$ & $4(17)$ & 0.841 \\
\hline \multicolumn{6}{|l|}{ Location } \\
\hline SB : colon : SB + colon (\%) & $54: 3: 43$ & $54: 2: 44$ & $27: 0: 73$ & $65: 13: 22$ & 0.004 \\
\hline \multicolumn{6}{|l|}{ Operator } \\
\hline Non-CRS & $16(7)$ & $4(2)$ & $2(18)$ & $10(44)$ & $<0.001$ \\
\hline Non-IBD surgeon & $26(11)$ & $7(4)$ & $3(27)$ & $16(70)$ & $<0.001$ \\
\hline Stapled anastomosis & $212(91)$ & $198(99)$ & $9(82)$ & $5(22)$ & $<0.001$ \\
\hline Indication & Obstruction (33) & Obstruction (35) & Obstruction (36) & Peritonitis (35) & $<0.001$ \\
\hline Complication & $37(16)$ & $34(17)$ & $0(0)$ & $3(13)$ & 0.293 \\
\hline
\end{tabular}

Values are presented as number (\%) unless otherwise indicated.

SS, side-to-side; SE, side-to-end; EE, end-to-end; SB, small bowel; CRS, colorectal surgeon; IBD, inflammatory bowel disease. 
anastomosis, and were more often operated on by a general surgeon. No statistical differences in age, sex, complications, and reoperation rates were found among the groups.

When RFS outcomes were compared according to the type of anastomosis, no differences among the 3 groups (EE, ES, and SS) were observed ( $\mathrm{P}=0.051)$ (Fig. 1). However, the hand-sewn group had a lower reoperation rate than the staple group $(\mathrm{P}=$ 0.040) (Fig. 2). No significant differences among the 3 surgeon groups were noted (Fig. 3). Non-IBD and IBD surgeons were compared with respect to anastomosis type and material used. Out of 233 surgeries, 207 were performed by IBD surgeons. Staplers were used in 203 patients (95.8\%), of which 192 (96.5\%) uses were in an SS fashion $(\mathrm{P}<0.001)$. In the non-IBD surgeon group, 17 out of 26 (65.4\%) were hand-sewn anastomoses, with 16 reconstructions $(69.6 \%)$ having been done in an EE fashion ( $P$ $<0.001)$. General surgeons were compared to colorectal plus IBD surgeons. Of the 217 patients operated on by colorectal surgeons,

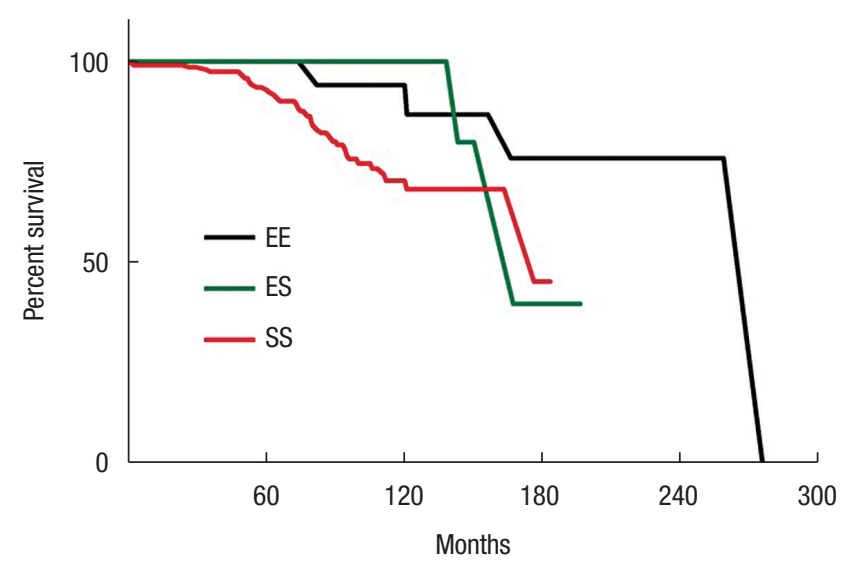

Fig. 1. Cumulative reoperation-free survival according to anastomosis configuration: side-to-side (SS), end-to-side (ES), and end-to-end (EE) anastomosis $(\mathrm{P}=0.051)$.

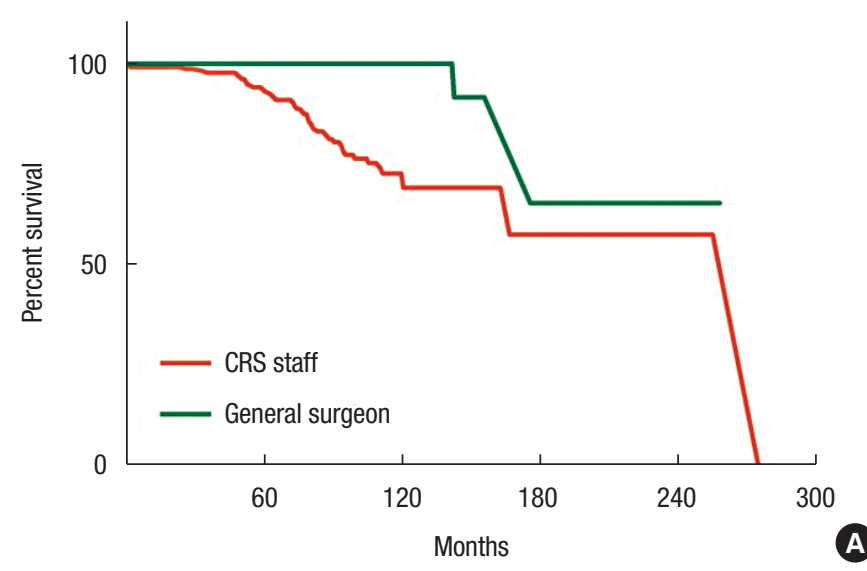

207 (95.4\%) were stapled, with 195 (89.9\%) having been done in an SS fashion. In the general surgeon group, a hand-sewn technique was used on 11 out of 16 patients $(68.8 \%)$, with 10 reconstructions having been done in an EE fashion $(\mathrm{P}<0.001)$.

In all, 50 patients (22\%) in our current series had reoperations after primary surgery, and the reoperation rates at 5 years, 10 years, and 15 years were $11.7 \%, 20.8 \%$, and $25.9 \%$, respectively. Of these 50 reoperations, anastomotic strictures were found in 12 patients $(24 \%)$. No differences in reoperation rate based on anastomosis configuration, anastomotic strictures were found in the EE group $(\mathrm{P}=0.036)$ (Table 2$)$.

\section{DISCUSSION}

Postoperative disease recurrence is common after CD surgery and may necessitate surgery and further bowel resections. Reducing short-term complications, recurrence, and reoperations is the

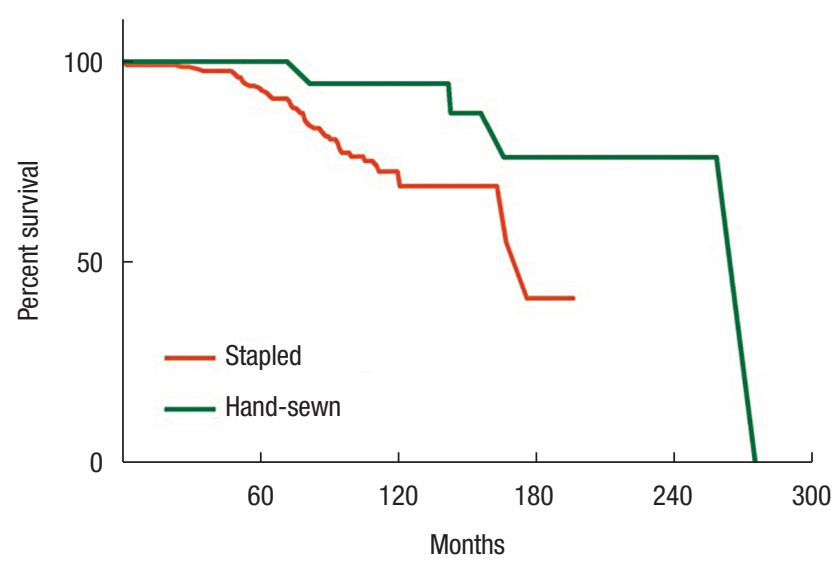

Fig. 2. Cumulative reoperation-free survival comparing anastomosis materials $(\mathrm{P}=0.040)$.

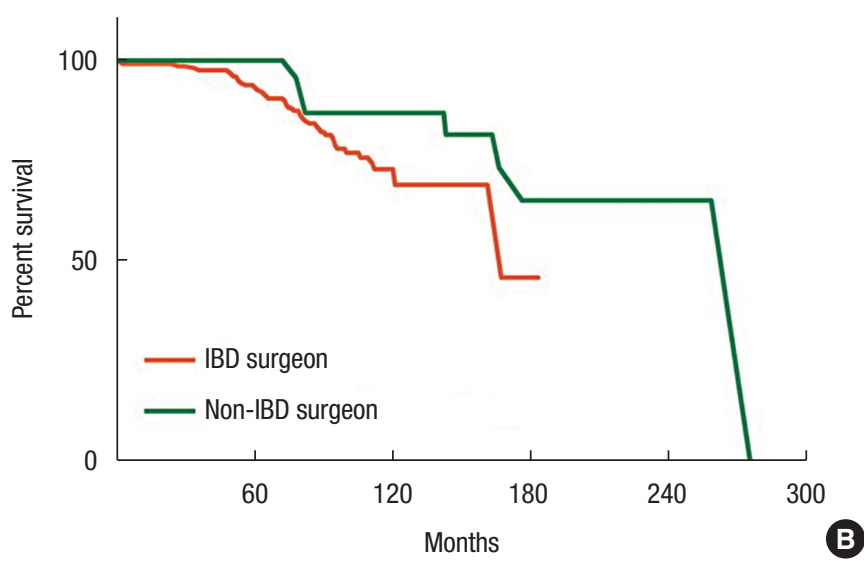

Fig. 3. Cumulative reoperation-free survival comparing colorectal surgeons (CRS) and general surgeons $(\mathrm{P}=0.106)(\mathrm{A})$ and inflammatory bowel disease (IBD) surgeons and non-IBD surgeons $(\mathrm{P}=0.138)(\mathrm{B})$. 
Table 2. Reoperations associated with anastomotic stricture.

\begin{tabular}{lrrrcc}
\hline & $\begin{array}{c}\text { Total } \\
(n=233)\end{array}$ & $\begin{array}{c}\text { SS } \\
(n=199)\end{array}$ & $\begin{array}{c}\text { SE } \\
(n=11)\end{array}$ & $\begin{array}{c}\text { EE } \\
(n=23)\end{array}$ & P-value \\
\hline Reoperation & $50(22)$ & $44(22)$ & $2(18)$ & $4(17)$ & 0.841 \\
$\begin{array}{c}\text { Anastomosis } \\
\text { involvement }\end{array}$ & $29 / 50(58)$ & $24 / 44(55)$ & $1 / 2(50)$ & $4(100)$ & 0.205 \\
$\begin{array}{c}\text { Anastomosis } \\
\text { stricture }\end{array}$ & $12 / 50(24)$ & $9 / 44(21)$ & $0 / 2(0)$ & $3(75)$ & 0.036 \\
\hline
\end{tabular}

Values are presented as number (\%).

SS, side-to-side; SE, side-to-end; EE, end-to-end.

most important concern of the treating surgeon. The percentages of disease recurrence requiring resurgery at 5- and 20-year intervals are, on average, $11 \%-32 \%$ and $46 \%-55 \%$, respectively [ 13 , 14]. In our current study, $22 \%$ of the patients were reoperated on and required further resection during the median follow-up of 7.8 years. The reoperation rates among the total 269 cases at 5-, 10-, and 15 -year intervals were $12 \%, 28 \%$, and $47 \%$, respectively, consistent with previous studies.

Several prognostic factors influencing the outcome of $\mathrm{CD}$ have been investigated to identify and reduce disease recurrence. The incidence of postoperative peri-anastomotic site recurrence in $\mathrm{CD}$ is above $90 \%$ [15]; this has generated interest in intraoperative strategies, such as changing the anastomosis configuration to reduce disease recurrence and complications. A stapling anastomosis is commonly used in clinical practice because staples are simple to use and require less time; they are also reliable and safe [16]. Among the surgeons who treated the patients in this study, 91\% preferred a stapling anastomosis to a hand-sewn anastomosis. No statistical differences were seen in terms of complications and RFS between the stapling and the hand-sewn groups. However, anastomosis site stricture was 3 times more common in the handsewn EE group than in the stapled SS group.

Many studies to date have favored stapled SS anastomoses, finding that they produce lower recurrence rates than hand-sewn $\mathrm{EE}$ anastomoses [7, 8, 17]. A claim has been made that SS anastomoses maintain better lateral blood flow and that the wide lumen prevents luminal stenosis and fecal pooling, in turn preventing early disease recurrence. Simillis et al. [18] found reduced anastomosis leakage in an SS group, but no significant differences in perianastomotic recurrence and reoperation related to perianastomotic recurrence. Scarpa et al. [19] reported that their stapled SS and hand-sewn SS anastomosis groups had a significantly lower reoperation rate than their stapled SE group, although the disease recurrence rates in the three groups were similar. These findings might explain the advantage of the SS configuration, over the other methods, in delaying reoperation by relieving symptomatic obstructions, irrespective of the material used for reconstruction.

A previous randomized control trial found that anastomosis type did not affect outcomes in terms of complications, leakage, and reoperation rate. SS and EE anastomoses were equally safe to perform, but stapler use did reduce the anastomosis time and overall duration of surgery [20]. Guo et al. [21] compared SS anastomoses (both hand-sewn and stapled) to hand-sewn EE, hand-sewn ES, stapled ES, and stapled EE anastomoses. They found no statistical superiority of any of the configurations in reducing endoscopic and symptomatic recurrence. However, lower endoscopic symptomatic recurrence was seen in the SS group. No significant differences were noted in terms of leak rate, reoperation, postoperative hospital stay, or complications.

Among the present study patients, the stapled SS anastomosis was the preferred configuration among colorectal and IBD surgeons. The conventional hand-sewn EE anastomosis had outcomes similar to those in the SS group, but was significantly associated with anastomotic site stricture. No statistical differences in terms of perianastomotic disease recurrence, other complications, or reoperation rate were noted. The use of a stapled SS anastomosis in CD surgery is not only safe for bowel reconstruction, it also helps delay reoperation for symptomatic perianastomotic recurrence [8]. We believe that the advantages of stapling procedures (they reduce operating time, are easy to perform, and result in minimal contamination, among others) can overcome their financial burden.

This retrospective study had limitations of strong selection bias and weighted grouping. The EE anastomosis was the method of restoring bowel continuity preferred by general surgeons during the early period of this study (before 2002). This included a small portion of patients and a majority of operations performed under emergency conditions. However, the other half (after 2002) included more patients and operations performed by a single IBD surgeon using a stapler as a priority method of bowel restoration. This uneven distribution of the cohort might have hindered the identification of the true benefits of the various anastomosis configurations.

In conclusion, stapling anastomosis is safe and reliable, and it reduces the operating time. However, hand-sewn and stapling techniques do not differ significantly in terms of complications, recurrence, and reoperation. Although the EE anastomosis has a higher risk of anastomosis site stricture, the type of anastomotic configuration may not influence reoperation after surgery in patients with $\mathrm{CD}$.

\section{CONFLICT OF INTEREST}

No potential conflict of interest relevant to this article was reported.

\section{REFERENCES}

1. Olaison G, Sjödahl R, Tagesson C. Glucocorticoid treatment in ileal Crohn's disease: relief of symptoms but not of endoscopically viewed inflammation. Gut 1990;31:325-8. 
2. Michelassi F, Balestracci T, Chappell R, Block GE. Primary and recurrent Crohn's disease. Experience with 1379 patients. Ann Surg 1991;214:230-8; discussion 238-40.

3. Goldberg PA, Wright JP, Gerber M, Claassen R. Incidence of surgical resection for Crohn's disease. Dis Colon Rectum 1993;36: 736-9.

4. Bernell O, Lapidus A, Hellers G. Risk factors for surgery and postoperative recurrence in Crohn's disease. Ann Surg 2000;231:38-45.

5. Borley NR, Mortensen NJ, Jewell DP. Preventing postoperative recurrence of Crohn's disease. Br J Surg 1997;84:1493-502.

6. Cottone M, Rosselli M, Orlando A, Oliva L, Puleo A, Cappello M, et al. Smoking habits and recurrence in Crohn's disease. Gastroenterology 1994;106:643-8.

7. Yamamoto T, Bain IM, Mylonakis E, Allan RN, Keighley MR. Stapled functional end-to-end anastomosis versus sutured endto-end anastomosis after ileocolonic resection in Crohn disease. Scand J Gastroenterol 1999;34:708-13.

8. Hashemi M, Novell JR, Lewis AA. Side-to-side stapled anastomosis may delay recurrence in Crohn's disease. Dis Colon Rectum 1998;41:1293-6.

9. Ikeuchi H, Kusunoki M, Yamamura T. Long-term results of stapled and hand-sewn anastomoses in patients with Crohn's disease. Dig Surg 2000;17:493-6.

10. Scott NA, Sue-Ling HM, Hughes LE. Anastomotic configuration does not affect recurrence of Crohn's disease after ileocolonic resection. Int J Colorectal Dis 1995;10:67-9.

11. Moskovitz D, McLeod RS, Greenberg GR, Cohen Z. Operative and environmental risk factors for recurrence of Crohn's disease. Int J Colorectal Dis 1999;14:224-6.

12. D’Haens GR, Geboes K, Peeters M, Baert F, Penninckx F, Rutgeerts P. Early lesions of recurrent Crohn's disease caused by infusion of intestinal contents in excluded ileum. Gastroenterology
1998;114:262-7.

13. Yamamoto T. Factors affecting recurrence after surgery for Crohn's disease. World J Gastroenterol 2005;11:3971-9.

14. Yamamoto T, Keighley MR. Stapled functional end-to-end anastomosis in Crohn's disease. Surg Today 1999;29:679-81.

15. Farmer RG, Hawk WA, Turnbull RB Jr. Clinical patterns in Crohn's disease: a statistical study of 615 cases. Gastroenterology 1975;68(4 Pt 1):627-35.

16. West of Scotland and Highland Anastomosis Study Group. Suturing or stapling in gastrointestinal surgery: a prospective randomized study. Br J Surg 1991;78:337-41.

17. Muñoz-Juárez M, Yamamoto T, Wolff BG, Keighley MR. Widelumen stapled anastomosis vs. conventional end-to-end anastomosis in the treatment of Crohn's disease. Dis Colon Rectum 2001;44:20-5; discussion 25-6.

18. Simillis C, Purkayastha S, Yamamoto T, Strong SA, Darzi AW, Tekkis PP. A meta-analysis comparing conventional end-to-end anastomosis vs. other anastomotic configurations after resection in Crohn's disease. Dis Colon Rectum 2007;50:1674-87.

19. Scarpa M, Angriman I, Barollo M, Polese L, Ruffolo C, Bertin M, et al. Role of stapled and hand-sewn anastomoses in recurrence of Crohn's disease. Hepatogastroenterology 2004;51:1053-7.

20. McLeod RS, Wolff BG, Ross S, Parkes R, McKenzie M; Investigators of the CAST Trial. Recurrence of Crohn's disease after ileocolic resection is not affected by anastomotic type: results of a multicenter, randomized, controlled trial. Dis Colon Rectum 2009;52: 919-27.

21. Guo Z, Li Y, Zhu W, Gong J, Li N, Li J. Comparing outcomes between side-to-side anastomosis and other anastomotic configurations after intestinal resection for patients with Crohn's disease: a meta-analysis. World J Surg 2013;37:893-901. 